\title{
SPECTROSCOPIC DIAGNOSTICS FOR IONS OBSERVED IN SOLAR AND COSMIC PLASMAS
}

\author{
Helen E Mason \\ Department of Applied Mathematics and Theoretical \\ Physics, Silver Street, Cambridge, CB3 9EW, U. K.
}

\begin{abstract}
The $X$-ray wavelength region $(1-200 \AA)$ is rich in spectral lines from highly ionised systems. Spectra from the solar atmosphere have been studied extensively with various instruments covering different wavelength regions. In this paper, we discuss the solar spectral line emission with particular reference to iron ions and helium like ions observed during solar flares. The atomic processes involved in the calculation of theoretical intensities for low density plasmas are outlined together with the diagnostic properties of the emission lines. Comparisons are made with available cosmic $X$-ray spectra and predicted spectra for future projects, such as AXAF.
\end{abstract}

\section{SOLAR ATMOSPHERE}

The solar corona has a characteristic temperature of around $2 \times 10^{6} \mathrm{~K}$ and a number density of about $10^{8} \mathrm{~cm}^{-3}$. This is in sharp contrast to the visible surface of the sun (photosphere) which has a temperature of $6000^{\circ} \mathrm{K}$ and a number density of $10^{17} \mathrm{~cm}^{-3}$. There is a steep transition region between the chromosphere and corona as illugtrated in figure 1. X-ray and UV images show that the distribution of material in the solar atmosphere is far from homogeneous indeed it is determined by the magnetic field configurations. Regions of open magnetic fields (coronal holes), which are believed to be the source of the solar wind, have very little coronal emission, whereas active regions show complex loop structure of closed magnetic fields. Small bipolar regions, bright points of $X-r a y$ emission, are also evident. These phenomena were studied extensively by instruments on Skylab - Apollo Telescope Mount (ATM)(1972). When the magnetic structures in active regions re-configure, vast amounts of energy are released (solar flares). The temperature in these explosive events rises to over $10^{7} \mathrm{~K}$ and the electron number density exceeds $10^{12} \mathrm{~cm}^{-3}$. The Solar Maximum Mission (SMM), launched in 1980, was designed specifically to study solar flares.

The higher the spatial resolution, the more complex are the structures which are observed. The HRTS instrument flown on Spacelab II (1985) obtained a spectral resolution better 
than 1". This instrument obtained spectra along a stigmatic slit from 1000-1800\&. Observations of transition region ions (eg CIV) indicate spatial inhomogeneities, variations in line width and shifts which dispel all notions of a homogeneous spherically symmetric transition region.

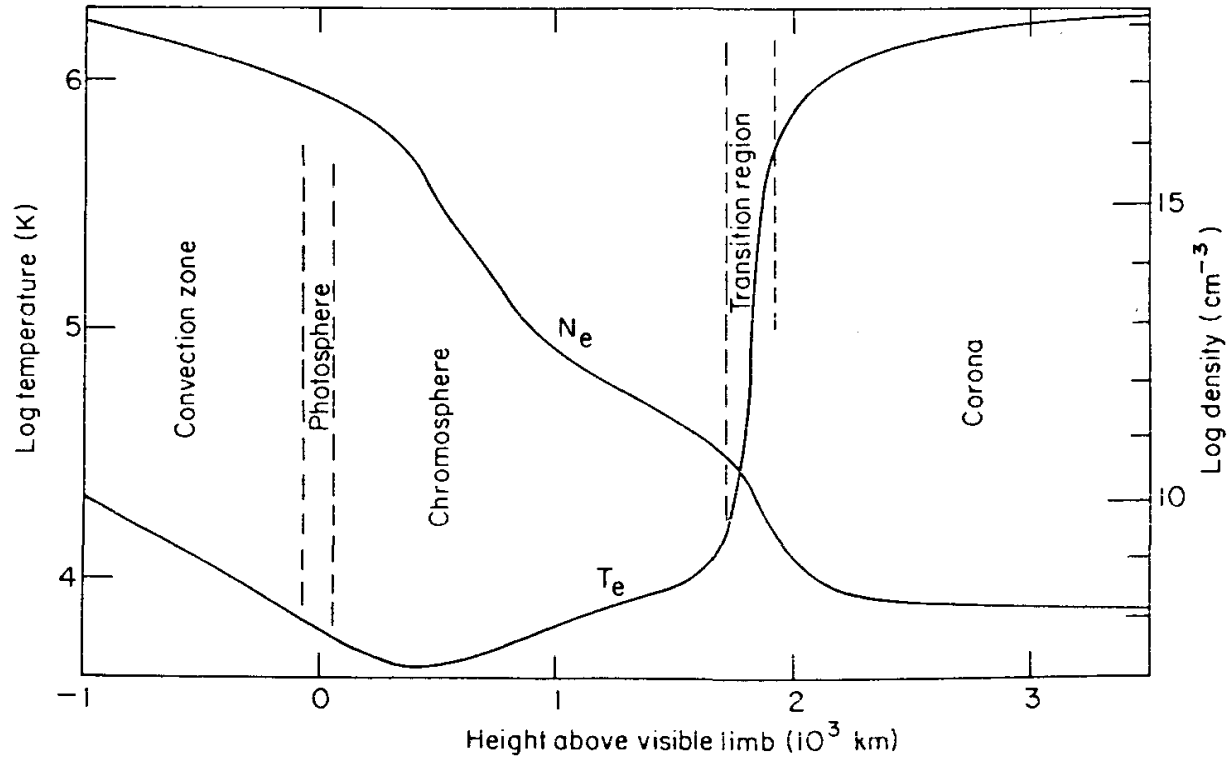

Figure 1 Schematic representation of the variation with height of the mean values of temperature and density in the outer layers of the sun (Gabriel and Mason, 1982).

\section{SPECTRAL LINE EMISSIVITY}

The line emissivity (per unit volume, per unit time) is

$$
E\left(\lambda_{i j}\right)=N_{j} A_{j i} \frac{h c}{\lambda_{i j}}
$$

where $A_{j i}$ - spontaneous radiative transition probability

h - Planck's constant

c - velocity of light

$\lambda i j$ - wavelength for transition $i-j$

$\mathrm{N}_{j}$ is the number density of level $\mathrm{j}$

$$
N_{j}\left(X^{+m}\right)=\frac{N}{N} j \frac{\left(X^{+m}\right)}{\left.X^{+m}\right)} \frac{N\left(X^{+m}\right)}{N(X)} \frac{N(X)}{N(H)} \frac{N(H)}{N_{e}} N_{e}
$$

$\frac{N}{N} j \frac{\left(x^{+m}\right)}{\left.x^{+m}\right)}$

- population of level j relative to the total number density of the ion $x^{+m}$. This is a function of electron density and temperature. 


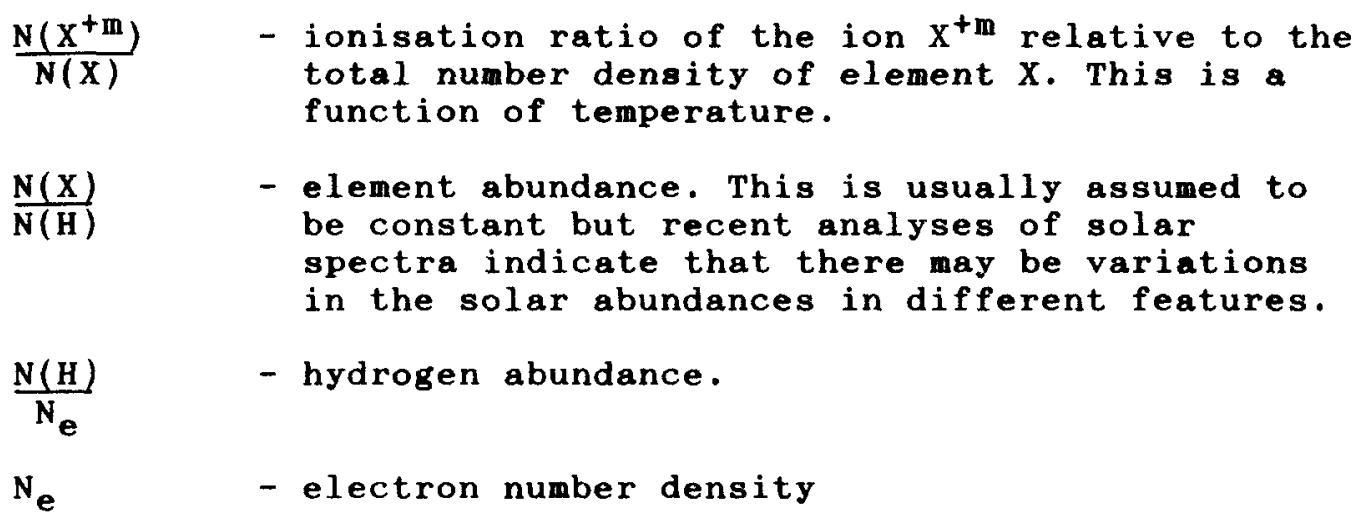

In most solar analyses we assume that the ionisation/recombination processes can be de-coupled from the level population calculations for individual ions. The intensity of a spectral line is then given by the integration of the line emissivity over the volume.

$$
I(\lambda i j)=\int_{V} E(\lambda i j) d V
$$

For the simple two level ion in coronal equilibrium, the population of the upper level $(j)$ is negligible compared to that of the ground level (i) $\left(N_{i}\left(X^{+m}\right) / N\left(X^{+m}\right) \approx 1\right)$. The upper level is excited by electron collisional excitation and de-excited by radiative decay.

$$
N_{i} N_{e} C_{i j}=N_{j} A_{j i}
$$

where $\mathrm{C}_{i j}$ is the electron excitation rate coefficient, which is the integration of the electron excitation crosssection over a Maxwellian velocity distribution, and $A_{j i}$ is the radiative transition probability.

$$
\text { Thus } \begin{aligned}
\epsilon\left(\lambda_{i j}\right) & =\frac{N}{N} \frac{\left(X^{+m}\right)}{\left.X^{+i n}\right)} \frac{N\left(X^{+m}\right)}{N(X)} \frac{N(X)}{N(H)} C_{i j} \frac{h c}{\lambda_{i j}} N_{e}^{2} \\
\epsilon\left(\lambda_{i j}\right) & =G(T) N_{e}^{2} \\
I\left(\lambda_{i j}\right) & =\int_{V} G(T) N^{2} d V
\end{aligned}
$$

This can be re-written in the form

$$
I(\lambda i j)=\int_{T} G(T) \phi(T) d T
$$


where $\varnothing(T)$ is called the differential emission measure which indicates the distribution of material as a function of temperature.

If the radiative decay rate is small, eg for forbidden or inter-system transitions, then other de-populating processes (eg electron collisions) compete and the ion departs from coronal equilibrium. The population of the upper ("metastable") level can become comparable to that of the ground level. At high densities the intensity of the line emission from the metastable level is proportional to $\mathrm{N}_{e}$ rather than $\mathrm{N}_{\mathrm{e}}{ }^{2}$. The ratio of this spectral line relative to an allowed line can be used as a density diagnostic (see figure 2). When the population of the metastable level is significant other levels can be excited from it as well as from the ground level. This again produces an electron density diagnostic line ratio. Once departures from coronal equilibrium occur, it is necessary to solve the level population equations including all relevant excitation and de-excitation processes to determine theoretical line intensities as a function of temperature and density.
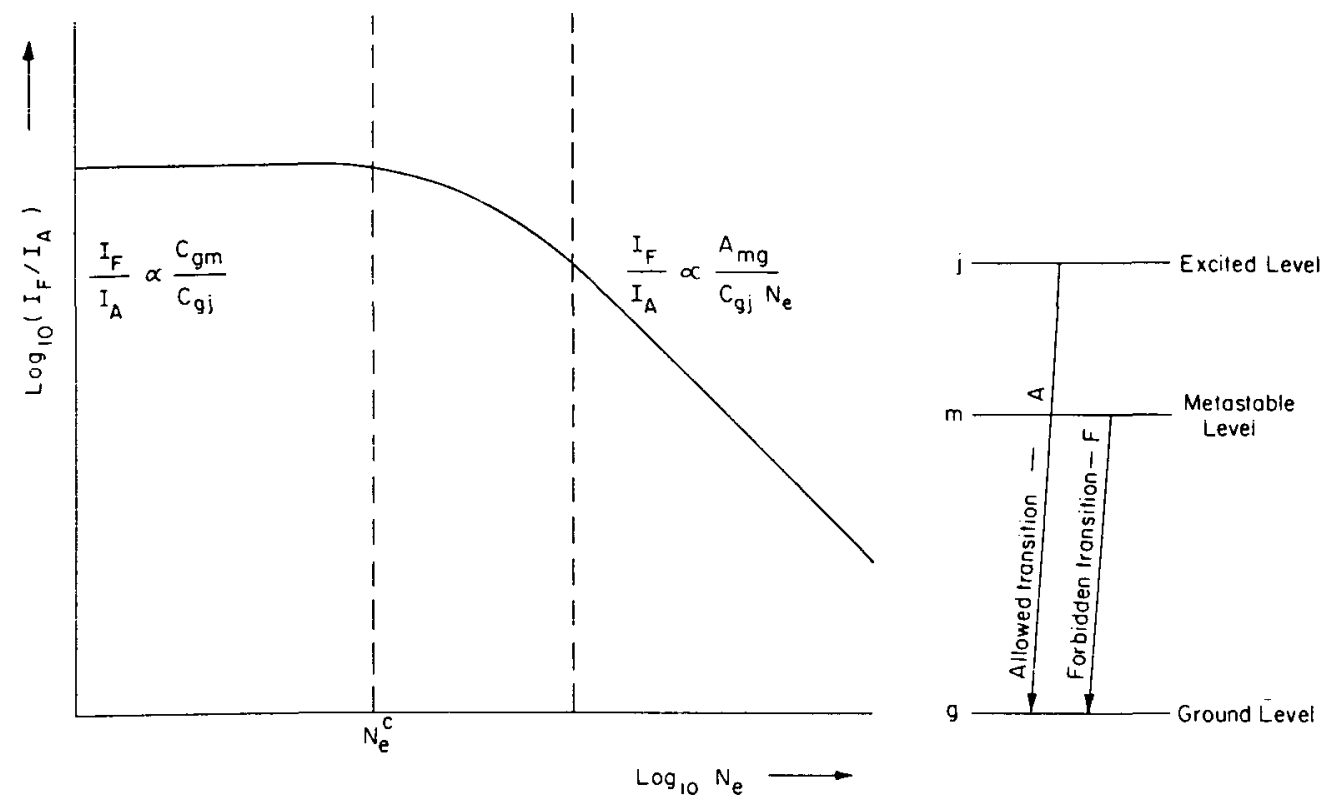

Figure 2 The variation with density of the intensity ratio of a forbidden to an allowed transition (Gabriel and Mason, 1982).

Spectroscopic diagnostics involve the analyses of one or more spectral lines to determine the characteristics of the emitting plasma. From a single line one can measure the line shape, width and shift or asymmetry. These parameters 
are determined by the ion temperature, any micro-turbulence, flows, waves etc. The absolute line intensity is a function of the electron temperature, density and the element abundance. The relative intensities of two or more 1 ines can be used to determine the electron density, temperature and equilibrium state of the plasma. The lines used must be carefully selected. There have been numerous reviews of spectroscopic diagnostics for the solar atmosphere eg Dere and Mason (1981), Gabriel and Mason (1982), Doschek (1985), Mason (1988). In this review paper we discuss the wavelength region 1 - $200 \AA$ giving a few specific examples.

\section{HIGHLY IONISED IRON IONS}

During solar flares, we see a whole sequence of spectral lines from highly ionised iron ions (FeXVIII FeXXVI). These fall into different wavelength regions. Take for example FeXXI $\left(\mathrm{T}_{\mathrm{e}}(\max ) \approx 10^{7} \mathrm{~K}\right)$, the transitions between $1 \mathrm{~s}^{2} 2 \mathrm{~s}^{2} 2 \mathrm{p}$ and $1 \mathrm{~s}^{2} 2 \mathrm{~s}^{2} \mathrm{p}^{3}$ fall between 90 and $150 \AA$, between $1 \mathrm{~s}^{2} 2 \mathrm{~s}^{2} 2 \mathrm{p}$ and $1 \mathrm{~s}^{2} 2 \mathrm{~s}^{2} 2 \mathrm{p}_{3} \mathrm{~d}$ fall around $12 \AA$, and between $1 \mathrm{~s}^{2} 2 \mathrm{~s}^{2} 2 \mathrm{p}$ and $1 \mathrm{~s}^{2} \mathrm{~s}^{2} 2 \mathrm{p}^{3}$ fall around $1.9 \AA$. Theoretical calculations for FeXXI were published by Mason et al (1979).

Only one set of flare spectra have been obtained in the EUV wavelength region. These data were obtained by Kastner et al (1974) with the osO-5 satellite (figure 3 ). This spectral range which has great diagnostic potential (see Mason et al, 1984) has been sadly neglected. In figure 4 is shown a spectrum of Capella obtained by the transmission grating spectrometer on EXOSAT. Some of the highly ionised iron spectral features are clearly resolved.

Figure 5 shows a solar flare spectrum in the $X-r a y$ wavelength region which was obtained by Neupert et al (1973). This can be compared with the $X$-ray spectrum of the Puppis A (figure 6) measured with the Focal Plane Crystal Spectrometer on the Einstein observatory. Several higher resolution solar flare spectra are now available, the best being that obtained by Phillips et al (1982) with the SMM XRP Flat Crystal spectrometer.

The Ka transitions around $1.9 \AA$, together with the satellite lines of FeXIV, have been extensively observed by SMM-XRP, SOLFLEX, and HINATORI instruments. Sample spectra are shown in figure 7 .

Many of these spectral lines can be used as diagnostics for electron density or temperature (see for example Gabriel and Mason, 1982). 


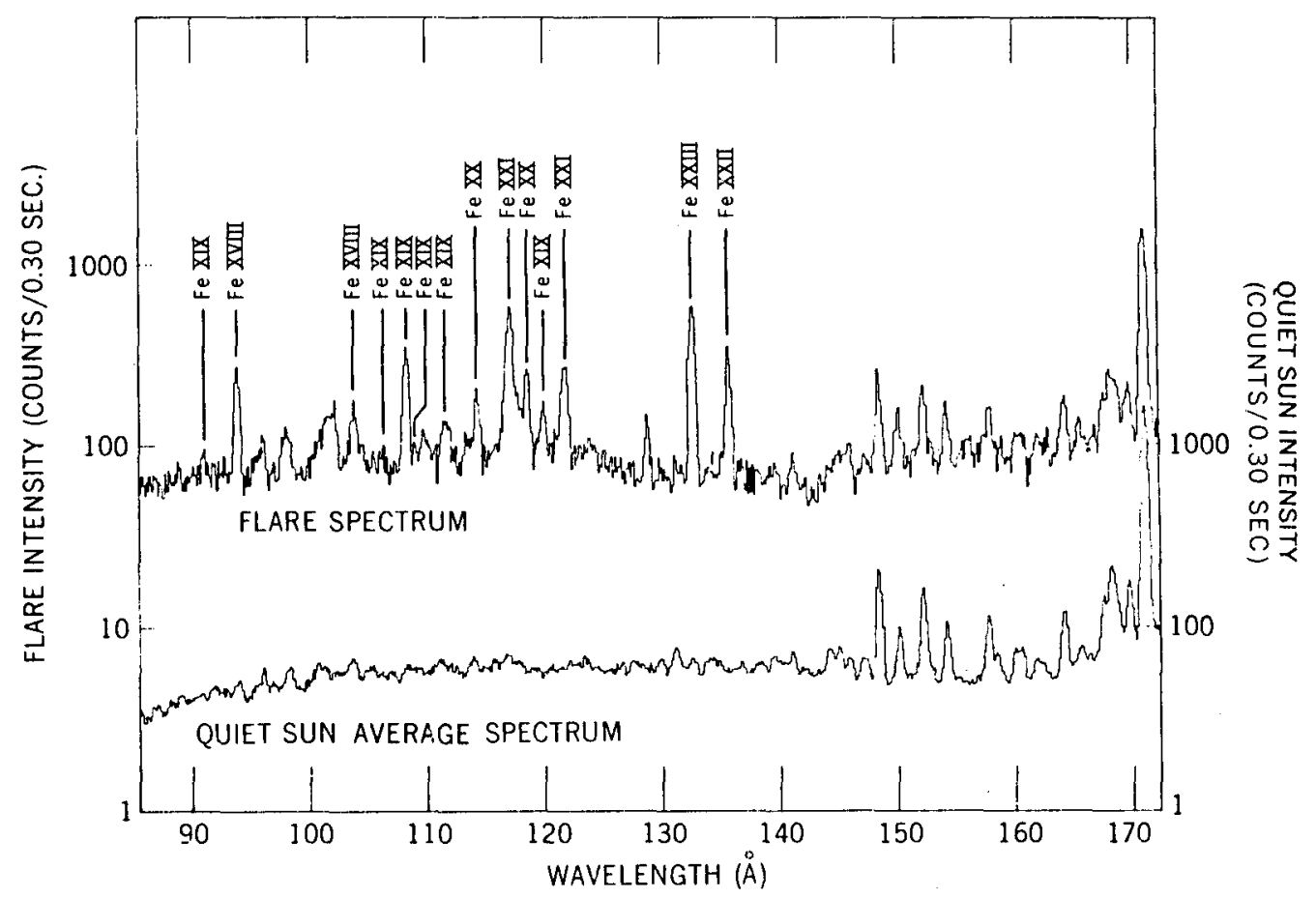

Figure 3 OSO-5 solar flare spectrum of emission lines from highly ionised iron ions (Kastner et al, 1974).

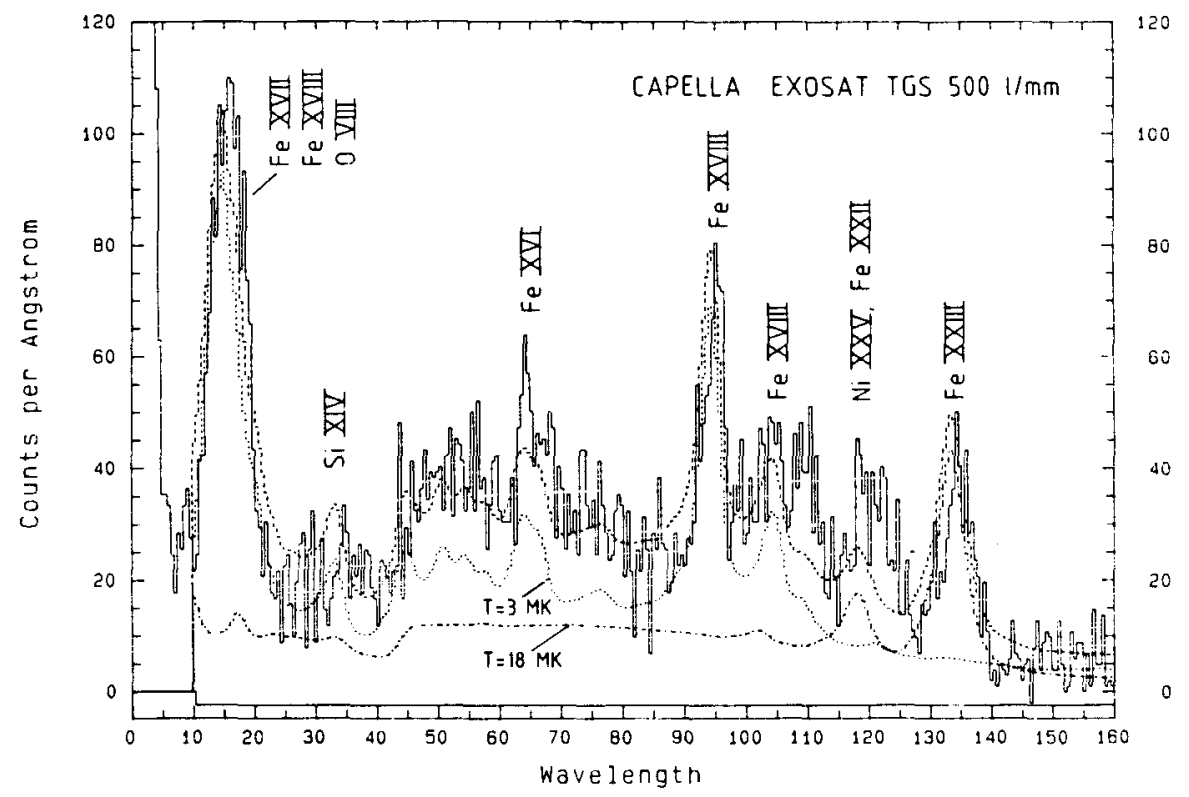

Figure 4 EXOSAT (500 1/mm) Transmission Grating spectrum of Capella (Brinkman et al, 1987). 


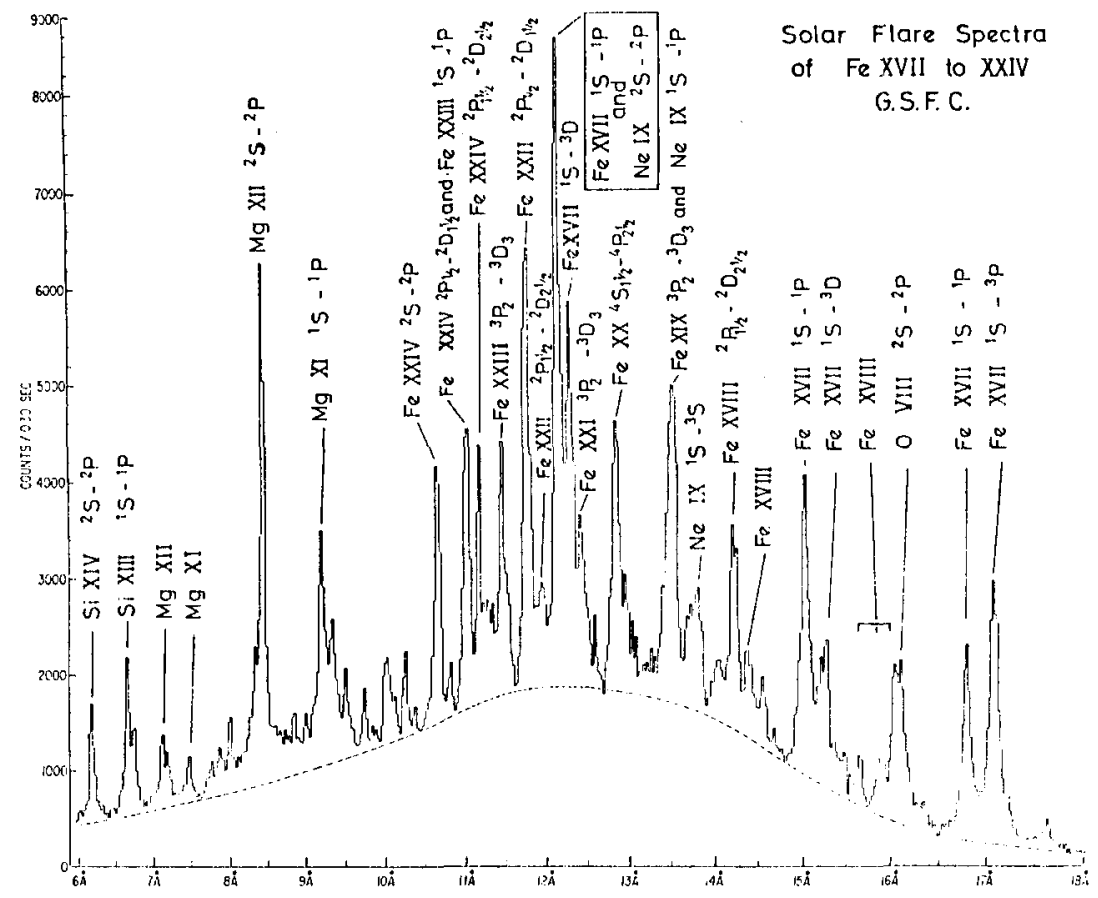

Figure 5 Solar flare spectrum from OSO-5 (Neupert et al, 1973).

EINSTEIN OBSERVATORY

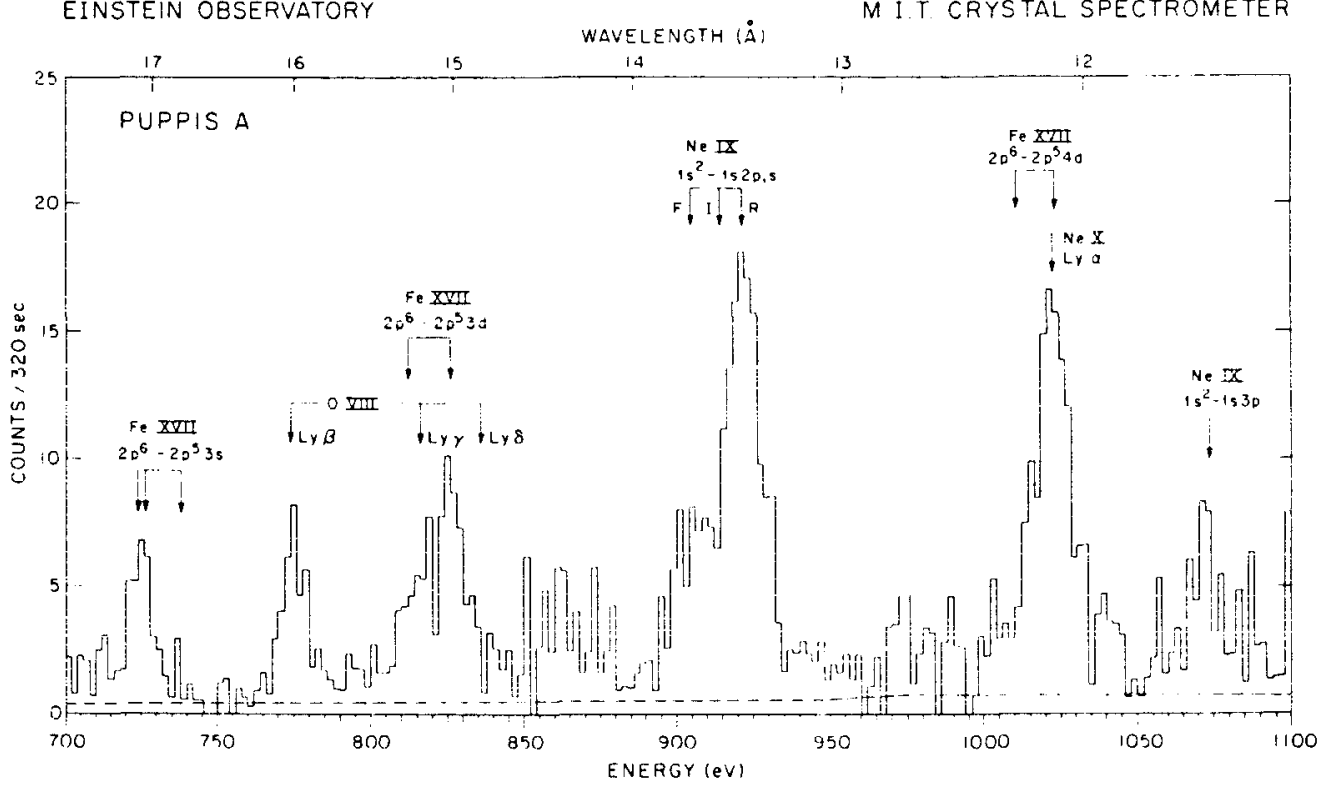

Figure 6. X-ray spectrum of Puppis A measured with the Focal Plane Crystal spectrometer on the Einstein Observatory (Winkler et al, 1981). 


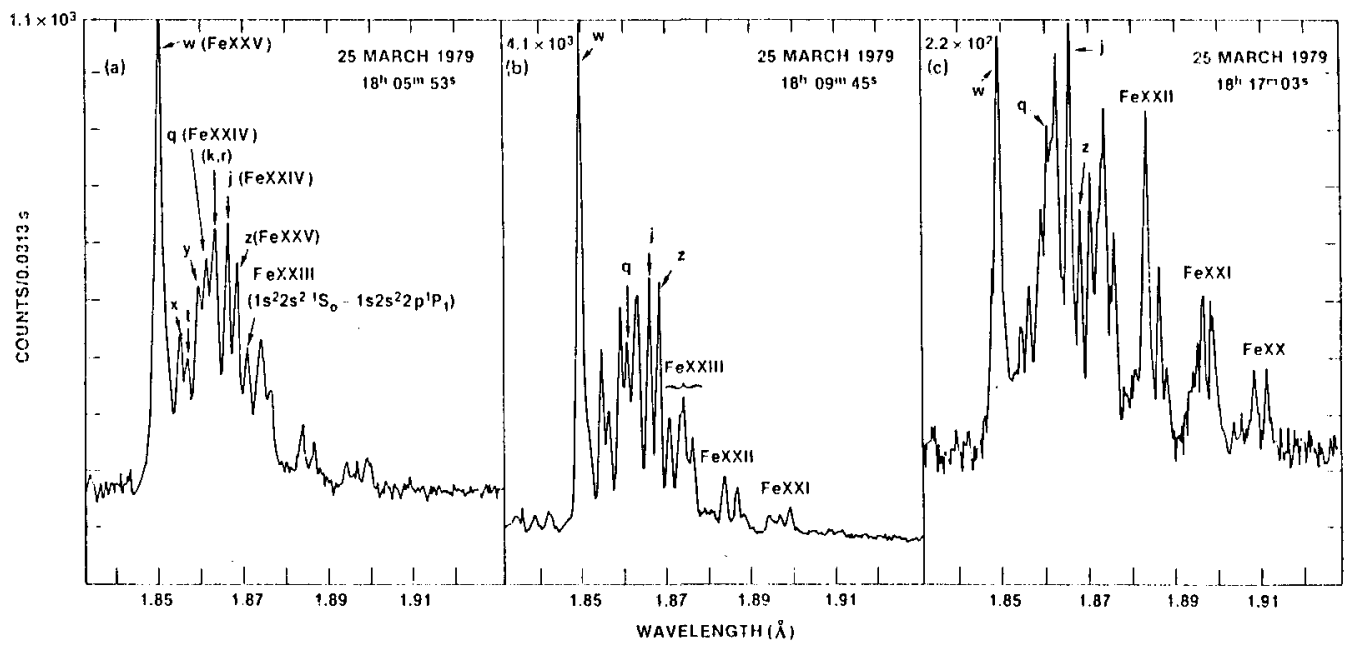

Figure 7 Flare spectra of iron line emission near $1.86 \AA$ obtained with the Naval Research Laboratory's Xray crystal spectrometer SOLFLEX (Doschek et al, $1979)$.

\section{HELIUM LIKE IONS}

The ratio $\mathrm{R}$ of the forbidden to the intercombination line from helium like ions can be used to determine electron density (see Gabriel and Mason, 1982). The lines from OVII were studied using the sOLEX instrument and electron densities were derived for solar flares (McKenzie et al, 1980). The NeIX lines observed with SMM-XRP have also been used to obtain electron density variations for solar flares (Wolfson et al, 1983). Unfortunately the NeIX lines which lie around $13 \AA$ are contaminated by FeXIX lines. However, it is possible to separate the two sets of lines using theoretical intensity ratios (Bhatia et al, 1989). Several helium like ions were studied by Brown et al (1986) from solar flare spectra obtained with a grazing incidence spectrograph $(10-95 \AA)$ aboard a sounding rocket.

\section{THEORETICAL CALCULATIONS}

The theoretical intensities of spectral lines depend on many atomic parameters. It is necessary to solve the atomic structure and electron scattering problems to obtain radiative data and collision rates. Many such calculations have been carried out by the author and colleagues. A programme of work is in progress in Dr A Burgess' group at 
Cambridge to calculate and assess available atomic data for astrophysical applications (Burgess et al, 1988, 1989). An atomic data bank is being compiled for input into analysis programs such as time dependant ionisation calculations and differential emission measure analyses. These are of special relevance to future solar and astrophysical missions, particularly those discussed in this conference.

\section{REFERENCES}

Bhatia, A. K., Fawcett, B. C., Lemen, J. R., Mason, H. E., and Phillips, K. J. H., 1989, M.N.R.A.S., submitted.

Brinkman, A. C., VanRooijen, J. J., Bleeker, J. A. M., Dijkstru, J. H., Heise, J., deKorte, P. A. J., Mewe, R., and Paerels, F., 1987, Astro. Lett. and Commun., 26, 73. Brown, W. A., Bruner, M. E., Acton, L. W., and Mason H. E., 1986, Ap. J., 301, 981 .

Burgess, A., Mason, H. E., and Tully, J. A., 1988, J de Phys., 49, C1-107.

Burgess, A., Mason, H. E., and Tully, J. A., 1989, Astr. Ap., in press.

Dere, K. P., and Mason, H. E., 1981, in Solar Active Regions, ed F. Orrall (Boulder:Colorado Associated University Press) chap. 6 .

Doschek, G. A., 1985, Autoionisation, ed A. Tenkin, chap. 6 . Doschek, G. A., Kreplin, R. W., and Feldman, U., 1979, Ap. $J$. Lett., 233, 457 .

Gabriel, A. H., and Mason, H. E., 1982, in Applied Atomic Collision Physics, Vol. 1, ed. H. S. W. Massey, E. W. McDaniel, and B. Bederson (New York:Academic), p. 345 . Mason, H. E., 1988, J. de Phys., 49, C1-13.

Mason, H. E., Bhatia, A. K., Kastner, S. O., Neupert, W. M., and Swartz, M., 1984, Solar Phys., 92, 199.

Mason, H. E., Doschek, G. A., Feldman, U., and Bhatia, A. K., 1979, Astr. Ap., 73, 74 .

Kastner, S. O., Neupert, W. M., Swartz, M., 1974, Ap. J., 191,26 .

McKenzie, D. L., Broussard, R. M. , Landecker, P. B., Rugge, H. R., Young, R. M., Doschek. G. A., and Feldman, U., 1980, Ap. J., 238, L43.

Neupert, W. M., Swartz, M., and Kastner, S. O., 1973, Solar Phys., 31, 171 .

Phillips, K. J. H., Leibacher, J. W., Wolfson, C. J., Parkinson, J. H., Fawcett, B. C., Kent, B. J., Mason, H. E., Acton, L. W., Culhane, J. L., and Gabriel, A. H., $1982, A p . J ., 256,774$.

Winkler, P. F., Canizares, C. R., Clark, G. W., Markert, T. H., Kalata, K., and Schnopper, H. W., 1981, Ap. J. Lett., $246, \mathrm{~L} 27$.

Wolfson, C. J., Doyle, J. G., Leibacher, J. W., and Phillips, K. J. H., 1983, Ap. J., 269, 319. 


\section{DISCUSSION-H. Mason}

Y. Gnedin: You showed you last slide with fine structure of a C IV region. Could you explain the spatial resolution of this region?

H. Mason: The spatial resolution achieved by the HRTS instrument is about $1^{\prime \prime}$. The structures seen are close to the spatial resolution of the instrument. Detailed studies of these structures have been carried out by Brueckner, Cook and Derel at the Naval Research Laboratory.

o. Vilhu: Have you ever observed any thermal or non-thermal velocities (asymmetries, red and blue shifts) during solar X-ray flares?

H. Mason: Non-thermal velocities have been observed during solar flares. In the early stages, the spectral lines are broad with blue shifted components. A lot of work has been done on this phenomena with the Ca XIX lines $(\sim 3 \AA)$ studied by SMM-XRP. The origin could be chromospheric evaporation from the foot points of the flare loops. Blueshifts in the Fe XXI (1354.1 $\AA$ ) line have also been studied with SMM-UVSP (Mason et al, 1986, Ap.J. 309, 435).

R. Pallavicini: As you know, XMM will try to measure densities using density sensitive line ratios in the spectral region $\sim 5$ to $50 \AA$. Besides OVIII, do you think there are appropriate lines - especially higher temperature lines - in that region which are suitable for deriving electron densities?

H. Mason: There are several density sensitive ratios in this wavelength region A review is given in Brown et al (1986, Ap.J. 301, 981).

H. Gursky: Could you comment on the question I asked Dr. Raymond; namely, the effect of purely plasma physics effects on the calculations that you present?

H. Mason: In "coronal equilibrium" it is usually assumed that the ionization/recombination and excitation/ de-excitation processes can be treated separately. If the timescale for changes in the plasmas parameters are faster than the timescale for atomic processes, it is necessary to couple the ionization/recombination and level population calculations. The collisional excitation and ionization rates etc. are usually calculated assuming a Maxwellian velocity distribution. This is not necessarily a good assumption for all plasmas. Strong magnetic fields can also affect atomic processes.

P. DeKorte: What spectra resolution is needed to carry out diagnostics on He-like ions like Ne IX in conjunction with the presence of Fe XIX lines?

H. Mason: About 005-0.1 $\AA$. The Ne IX lines are contaminated by Fe XIX lines. Even the resolution achieved by SMM-XRP was not sufficient to separate these blends. However, the amount of FeXIX contributing to the NeIX lines could be estimated theoretically if a spectral resolution $\sim 0.05-0.1 \AA$ is achieved. This would resolve the strongest FeXIX lines 135. 04-13.520 $\AA$ from the NeIX resonance line (13.446 $\AA$ ), (Bhatia et al 1989 M.N.R.A.S, submitted). 